\title{
Agôn
}

9| 2021

Rater

\section{Le rater mieux, selon Robyn Orlin}

\section{Myriam Blœdé}

\section{(2) OpenEdition}

Journals

Édition électronique

URL : https://journals.openedition.org/agon/9090

DOI : $10.4000 /$ agon.9090

ISSN : 1961-8581

\section{Éditeur}

Association Agôn

\section{Référence électronique}

Myriam Blœdé, «Le rater mieux, selon Robyn Orlin », Agôn [En ligne], 9 | 2021, mis en ligne le 15 novembre 2021, consulté le 17 janvier 2022. URL : http://journals.openedition.org/agon/9090 ; DOI : https://doi.org/10.4000/agon.9090

Ce document a été généré automatiquement le 17 janvier 2022.

Association Agôn et les auteurs des articles 


\title{
Le rater mieux, selon Robyn Orlin
}

\author{
Myriam Blœdé
}

1 On postulera ici que, dans la formation et le parcours artistique de la chorégraphe sudafricaine Robyn Orlin, le rater, le ratage et les ratés, le rater, le ratage ou les ratés, dans leurs multiples déclinaisons - accident, échec, chute, déroute, danger mortel, impossibilité (atroce) ${ }^{1}$, incompréhension, conflit(s), aporie, entropie, chaos, balagan, etc. - constituent une expérience, pour ne pas dire l'expérience, fondamentale. Et que, chemin faisant et de fil en aiguille, le partage (sensible) de cette expérience est devenu le fondement de son théâtre.

2 Sans espérer ni prétendre en démontrer le pourquoi (cause et visée), on pointera (d'un doigt tremblant) une ou deux pistes, de possibles voies d'explication. Et on tentera plutôt de saisir comment, à quels niveaux et sous quelles formes, s'exprime, dans son théâtre, ce désir ou cette volonté de partage. Car dans «le rater mieux, selon Robyn Orlin ", l'inclusion est, on le verra, le maître mot. Aucune étape du processus de création, aucun moment de la représentation, aucun élément de la composition, aucun membre de la communauté provisoire que constitue l'œuvre ne sera a priori laissé pour compte. Nous nous intéresserons d'abord à l'artiste elle-même, aux ratés qui la fondent et aux symptômes qu'ils produisent dans son écriture. Puis, à partir de cas concrets, nous verrons comment et avec qui, partenaires, destinataires, s'opère le partage du rater.

3 Le sol mouvant sur lequel nous nous appuierons dans cette entreprise, nos sources et supports d'analyse sont hétérogènes et, pour une grande part, relèvent aussi de l'expérience : ce sont d'abord quelques-unes parmi les vingt-neuf «pièces » de Robyn Orlin que nous avons vues, parfois à plusieurs reprises et dans différents contextes, depuis 1999. Ce sont aussi les données que nous avons pu collecter dans les coulisses ou les laboratoires de création de certaines d'entre elles, en échangeant avec Robyn Orlin ou en suivant son travail, au cours de workshops ou de répétitions. Ce sont enfin diverses sources textuelles, sonores ou filmiques, relatives à son œuvre, publiées ou non, auxquelles nous avons eu accès ${ }^{2}$.

4 Si Robyn Orlin se situe résolument du côté de la pratique, si, autrement dit, elle se défend de théoriser sa pratique, elle se prête assez volontiers aux entretiens et 
rencontres avec le public. Sa parole, son discours, direct ou médiatisé, fait donc également partie des matériaux sur lesquels nous avons travaillé.

\title{
Who is Robyn Orlin?
}

\author{
LE CLIENT : Dieu a fait le monde en six jours, et \\ vous, vous n'êtes pas foutu de me faire un \\ pantalon en six mois. \\ LE TAILLEUR : Mais, monsieur, regardez le \\ monde, et regardez votre pantalon.
}

C'est ainsi que Beckett expose cette histoire bien connue, en épigraphe de "Le monde et le pantalon ", l'article qu'il consacrait en 1945 à la peinture (aux peintures, devraiton dire) de Bram et Geer van Velde. En matière de rater (c'est que Beckett, comme on sait, en connaît un rayon), ce dialogue est une réussite, un trésor d'éloquence et de concision - aussi, pourquoi se priver du plaisir de le citer, une fois encore. De là à supposer que Robyn Orlin pourrait se prendre pour Dieu, il y a un gouffre que l'on ne se hasardera pas à franchir. En l'occurrence, sa position serait plutôt celle du tailleur : faire des pantalons, encore et encore, sans jamais perdre de vue (pour essayer de compenser, peut-être) la malfaçon du monde (qui aura toujours besoin de pantalons). Mais, comme dirait Beckett encore : "Pour commencer, parlons d'autre chose (...) $)^{3}$." Par exemple, pour commencer : qui est donc cette «chorégraphe " sud-africaine qui nous arrive tout droit d'Afrique du Sud avec ses performers, acteurs, danseurs, chanteurs sud-africains ? Oui. "Qui est Robyn Orlin?», se demandera-t-on.

Fort opportunément, c'est le titre même, en anglais, de l'autoportrait filmé de Robyn Orlin qu'a réalisé Philippe Lainé ${ }^{4}$. Petite merveille de concision et d'efficacité, Who is Robyn Orlin $^{5}$ ? commence dans un éclat de rire, par ces mots de Robyn Orlin qu'on ne voit pas, mais dont on reconnait la voix (pour autant qu'on la connaisse, bien sûr) : " I don't know who I am... I don't know who I am. I don't know where I live. I don't know whether I make dance or theater, or art $^{6} . .$. » Cut. Deux, et en fait non, ce sont alors trois femmes qui revendiquent, oralement et gestuellement, dans une aimable bousculade et un jovial échange d'arguments, toute l'attention de la caméra, le premier plan sinon le monopole (de/dans l'image), et l'identité de Robyn Orlin : «I am Robyn Orlin, Ich bin Robyn Orlin, je suis Robyn Orlin, pas de problème.» La scène (mais non le film) se clôt dans l'irrésolution, malgré le K.O. technique (un éclat de rire, encore) qu'Ann Masina semble opérer sur ses deux partenaires et rivales, Toni Morkel et Melissa Madden Gray (dite Meow Meow).

Toni Morkel, Melissa Madden Gray et Ann Massina dans Who is Robyn Orlin ?

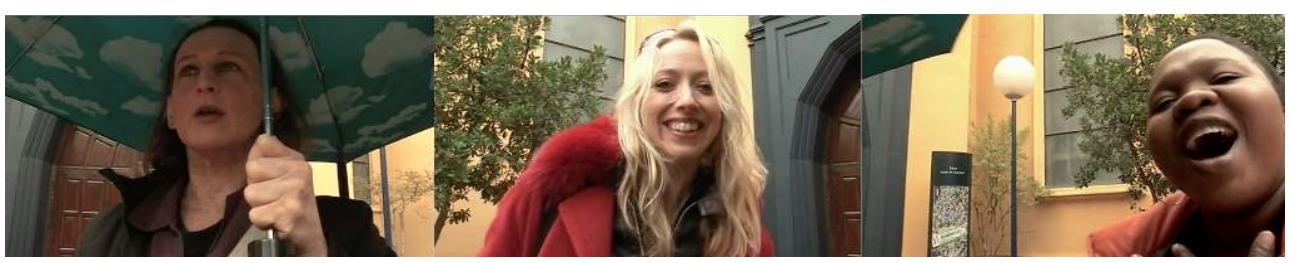

Photogrammes du film, réalisation Philippe Lainé

(c) Sweety Pie, 2011 
7 Au fond, la question de savoir qui est Robyn Orlin pourrait n'avoir guère d'importance (après tout, c'est son « œuvre " avec un grand $\mathbb{E}$, pas elle, que le spectateur vient voir). Pourtant, la poser fait d'emblée apparaître un point nodal, un motif véritable, dans l'écriture de la dame : constamment invoquée sur la scène de son théâtre, Robyn Orlin n'arrive jamais.

8 C'est le cas dans Daddy, I've seen this piece six times before and I still don't know why they're hurting each other... (Papa, j'ai déjà vu six fois cette pièce et je ne comprends toujours pas pourquoi ils se font du mal..., 1999) où, soit dit en passant, les ratés s'accumulent : le décor (des lais de papier kraft) s'effondre - manifestant ainsi sa présence -, Toni Morkel peine à monter, puis dérape sur le plateau, détruisant l'image qui vient d'y être patiemment composée, Gerard Bester ne parvient pas à ajuster son tutu... Et, malgré trois tentatives, Nelisiwe Xaba échoue dans l'exaltation d'un paradigme de la danse occidentale de tous les temps, la verticalité, représentée ici par la reprise d'une posture ou plutôt d'une action ordinaire, quotidienne et banale, surtout chez les femmes du Sud au Nord de l'Afrique, et qui consiste à se mouvoir, en l'occurrence à onduler, avec une charge sur la tête (successivement : un cygne en céramique, une bouteille d'1,5 1 d'eau, un régime de bananes). Où le rater intentionnel contribuerait à la charge critique de cette séquence, à l'égard des conventions de genre, esthétiques ou sociales notamment.

Nelisiwe Xaba dans Daddy..., Robyn Orlin, 1999

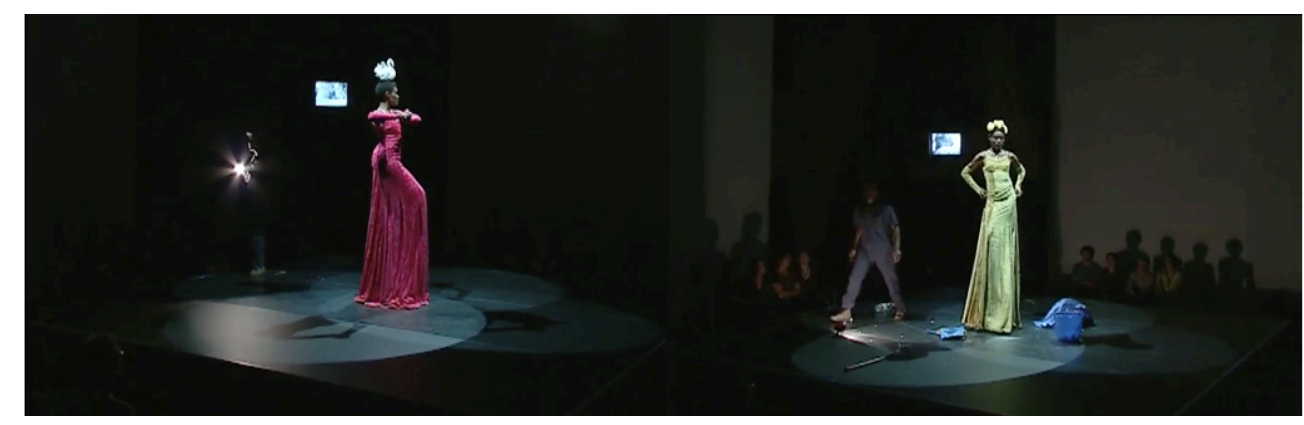

Photogrammes de la captation filmée au C ND, Pantin, le 5 novembre 2006

9 Mais surtout - et c'est là l'un des fils qui court dans la trame dramatique de Daddy... -, toutes ces tribulations et d'autres sont et ne sont que la conséquence d'une unique foirade: l'absence d'un personnage, nommé Robyn (Orlin), laquelle, comme on le comprend, représente l'auteure ${ }^{7}$, la chorégraphe, la metteuse en scène, la créatrice en un mot. Robyn est en retard, elle téléphone à trois reprises à l'un des acteurs qui se trouve sur la scène (Gerard Bester) pour s'en excuser, s'expliquer, rassurer, assurer qu'elle arrive, mais le temps passe, le public est là au complet, les performers et techniciens aussi ; et quelque part dans la salle se trouve sûrement le directeur du théâtre parmi d'autres programmateurs. Il se pourrait aussi, ou pas, que ce soir soit celui de la première du spectacle. Première mondiale, nationale ou hyper locale, peu importe (on connaît l'histoire du meilleur tailleur de la rue), dans tous les cas, l'enjeu est d'importance. Parce que c'est un impératif catégorique, the show must go on (d'ailleurs, cahin-caha, the show goes on), parce qu'aussi, selon l'un des protagonistes de la pièce ${ }^{8}$, The City Theater \& Dance Group (la compagnie de Robyn Orlin) est un ambassadeur, sur le plan culturel au moins, de cette "jeune démocratie " qu'est l'Afrique du Sud. Etc. etc. Mais Robyn ne vient pas. 
Telle est sans doute, sur fond de Nation arc-en-ciel, de vérité et de réconciliation, la fable de Daddy... le rendez-vous manqué de l'artiste avec son équipe, avec son public et avec les institutions (culturelles) dont elle dépend, celles qui lui garantissent les moyens d'œuvrer et de faire en sorte que, précisément, l'œuvre "produite », sinon l'artiste, rencontre «son " public .

\section{I don't know where I live... En l'absence de l'auteur}

11 En deçà de ce fiasco et pour en revenir à notre point de départ, ce que la question « qui est Robyn Orlin?» pointe aussi, et peut-être avant tout, est un raté originel, un malentendu, un scandale peut-être, dont pudiquement, sacrifiant aux conventions d'une certaine bienséance, on ne parle guère. Dont on ne parle pas, alors que de facto cette donnée première (et primaire aussi) crève les yeux. Alors, disons-le maintenant et passons à autre chose.

Lorsque Robyn Orlin arrive en France courant 1999, auréolée, pour Daddy... justement, d'un $3^{\mathrm{e}}$ prix aux Rencontres chorégraphiques de l'Afrique et de l'Océan indien d'Antananarivo (Madagascar, 1999), d'elle on sait seulement qu'elle est (sud-) africaine, ce qui de notre étroite fenêtre, suggère qu'elle est noire. Première déconvenue, premier paradoxe, première surprise en tous cas, cette Africaine-là, native de Johannesburg où elle vit le jour en 1955, est blanche. Bien sûr, nous n'ignorons pas que, depuis quelques siècles, des Blancs naissent en Afrique, en Afrique du Sud en particulier - sur son versant folklorique au moins, le fait est bien connu. Nous voilà contraints cependant d'ajuster notre vision et de prendre en compte un peu de cette sale histoire-là : ainsi, Robyn Orlin, c'est de la danse africaine blanche. Mais aussi : ainsi, Robyn Orlin fait partie de la catégorie dite, pudiquement, toujours, des « minorités visibles ».

Deux fois «raté », et même trois, si l'on tient compte du profil «est-européen ${ }^{10}$ » de cette artiste, sud-africaine de première génération, ses parents étant tous deux des juifs émigrés, respectivement de Pologne (sa mère, Rhoda Greene) et de Lituanie (son père, Barney Orlin) : malgré son engagement viscéral contre l'apartheid, voilà qui met Robyn du mauvais côté de la barrière, indifféremment celui des «minorités visibles » et celui des colons ${ }^{11}$ - et, par un effet-boomerang (ou miroir, ce qui, en l'occurrence, revient au même), malgré (ou à cause de) la bienveillance légèrement suspecte avec laquelle certains d'entre nous, pour qui l'Afrique reste un lointain, nous apprêtions à « recevoir » cette artiste africaine, voilà qui nous renvoie illico, assez brutalement, du même ou d'un autre (mauvais) côté, celui des lieux communs et des évidences fausses. Or il se trouve que, justement, les lieux communs et les évidences fausses travaillent les créations de Robyn Orlin.

Mais avant de passer à autre chose (tout en évitant de nous égarer sur la voie d'un quelconque ethnocentrisme ${ }^{12}$ ), notons tout de même que cette histoire de couleur de peau ou de Who is Robyn Orlin n'est pas sans conséquence : elle soulève la question du côté (bon ou mauvais), du camp (à choisir, si l'on peut), de la place (à occuper ou non)... À ce propos d'ailleurs, sans la prendre à son compte, Robyn Orlin évoquait un jour une problématique «typique des juifs sud-africains », ces " visiteurs ", ces déclassés, arrivés en Afrique du Sud au début du xxe siècle pour fuir les pogroms en Europe centrale et qui, sans être le moins du monde liés à ce pays, s'y retrouvaient aussitôt intégrés au 
premier rang de la société, sous le seul prétexte qu'ils étaient blancs ${ }^{13}$. Racialement assignés dans leur pays d'origine qu'ils décident de fuir, pour cette raison précise, ils se retrouvent, même si c'est d'une autre manière, racialement assignés dans leur pays d'accueil - et à nouveau, comme on l'a vu, si d'aventure ils s'aventurent sur leur continent d'origine ${ }^{14}$. Sur le même registre, mais dans un autre contexte, Robyn Orlin a expliqué qu'en Allemagne où elle vit actuellement, parce qu'elle n'est pas Allemande et parce qu'elle ne parlera jamais "assez correctement l'allemand", elle se vit comme « une citoyenne de $2^{\mathrm{e}}$ classe $»^{15}$.

Cette histoire, disions-nous, soulève la question de la place - celle qu'on occupe / celle qu'on décide d'assumer. Symétriquement, elle interroge le regard. Et la nécessité de l'ajuster. Ou, comme le disait l'autre, dialectiquement, elle interroge le «ce que nous voyons, ce qui nous regarde». Et peut-être, après tout... ce que nous voyons nous regarde : "Parler d'images dialectiques, c'est à tout le moins jeter un pont entre la double distance des sens (les sens sensoriels [...]) et celle des sens (les sens sémiotiques, avec leurs équivoques, leurs espacements propres). Or ce pont, ou ce lien (...) est originaire $(. . .)^{16} . »$

Mais surtout, ces conséquences prennent formes (dialectiquement) dans l'univers artistique de Robyn Orlin. Et elles se manifestent par tout un éventail de stratégies qui visent précisément à déplacer, dérouter, désorienter, divertir, dériver, inverser, renverser, tourner, détourner, retourner, disséminer... (le regard/son objet), et qui concourent à torpiller de manière quasi-systématique tout ce qui relève des conventions ou contribue à l'illusion du spectacle. Des stratégies qui, simultanément, en viennent parfois à parer celui-ci, jusqu'à l'écœurement, de tous les signes du spectaculaire ou du divertissement - car Robyn Orlin concède un goût très prononcé pour le kitsch et l'entertainment, ce qui déjà la pousse ou l'attire dans un certain champ du rater: selon les canons de la distinction intellectuelle et bourgeoise, le «mauvais goût », chez elle, n'est jamais loin. 


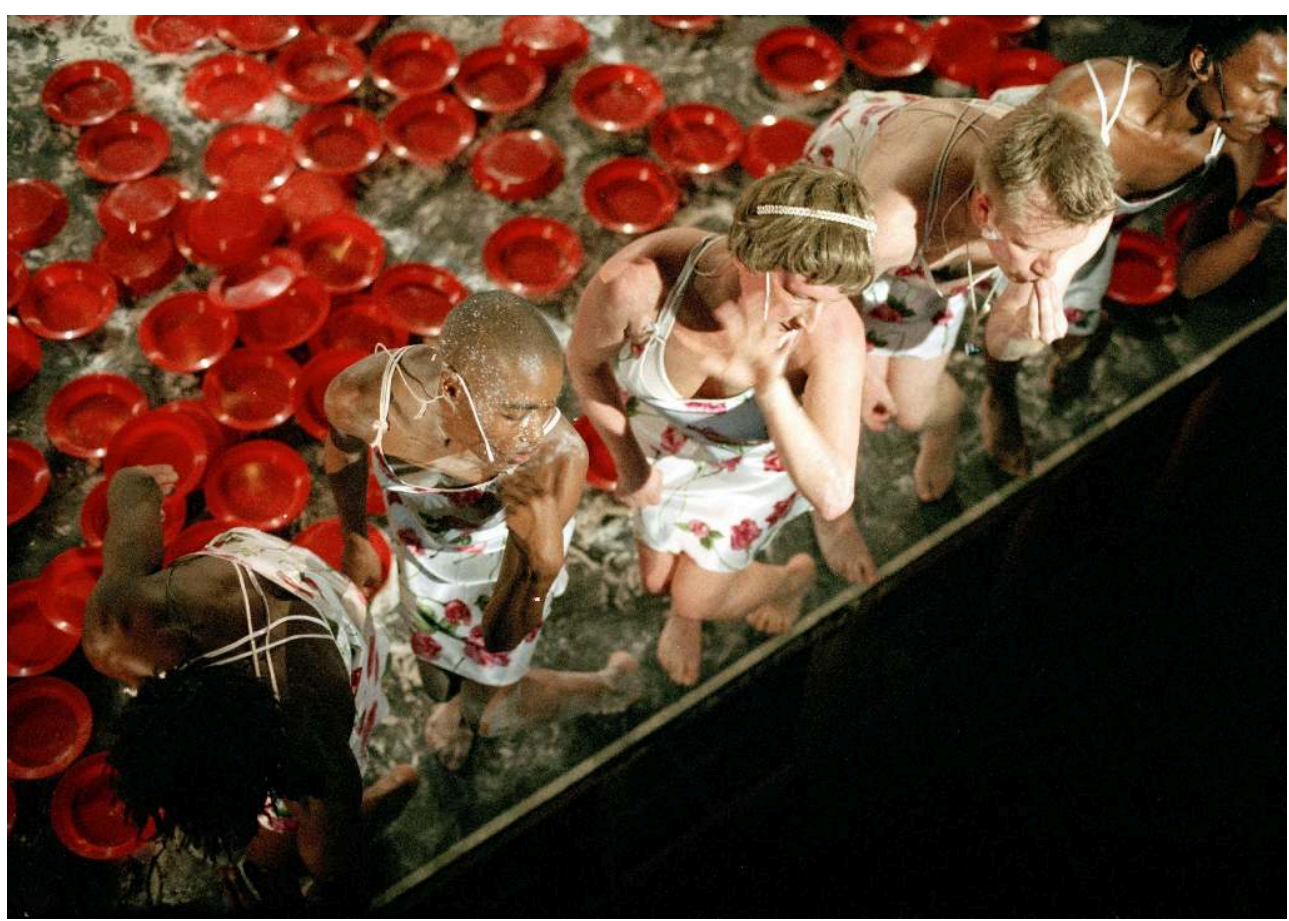

(c) John Hogg

Avec quelques variantes, où il apparaît que le personnage de Robyn n'est pas toujours seul en cause, la tragi-comédie de l'auteur absent est récurrente chez Robyn Orlin. Ainsi, dans je suis venu... j'ai vu... j'ai mâché des malabars... (2008) ${ }^{17}$, on le devine, c'est évidemment César qui manque à l'appel et que Seydou Boro se voit contraint de représenter au pied levé, même si, entre l'empereur Julius (Caesar) et le sculpteur César (Baldaccini), le doute subsiste. Dans Beauty remained for just a moment then returned gently to her starting position... (La beauté ne dura qu'un moment puis reprit doucement sa position initiale..., 2012), Dieu, c'est-à-dire God, que Teboho Letele appelle familièrement Godisto, est invoqué sur scène, mais Lui non plus ne viendra pas. Et c'est à nouveau Dieu, explicitement, qui est attendu en vain dans Hey Dude... I have talent... I'm just waiting for God... (Hé mec... j'ai du talent... je suis juste en train d'attendre Dieu..., 2005), God qui, dans la séquence d'ouverture de la pièce, deviendra Godot, puis Robyn - laquelle, nous dit-on, ne sait même pas tricoter, et le costume de Vera Mantero ne sera jamais prêt ${ }^{18}$... 
Vera Mantero dans Hey Dude..., Robyn Orlin, 2005

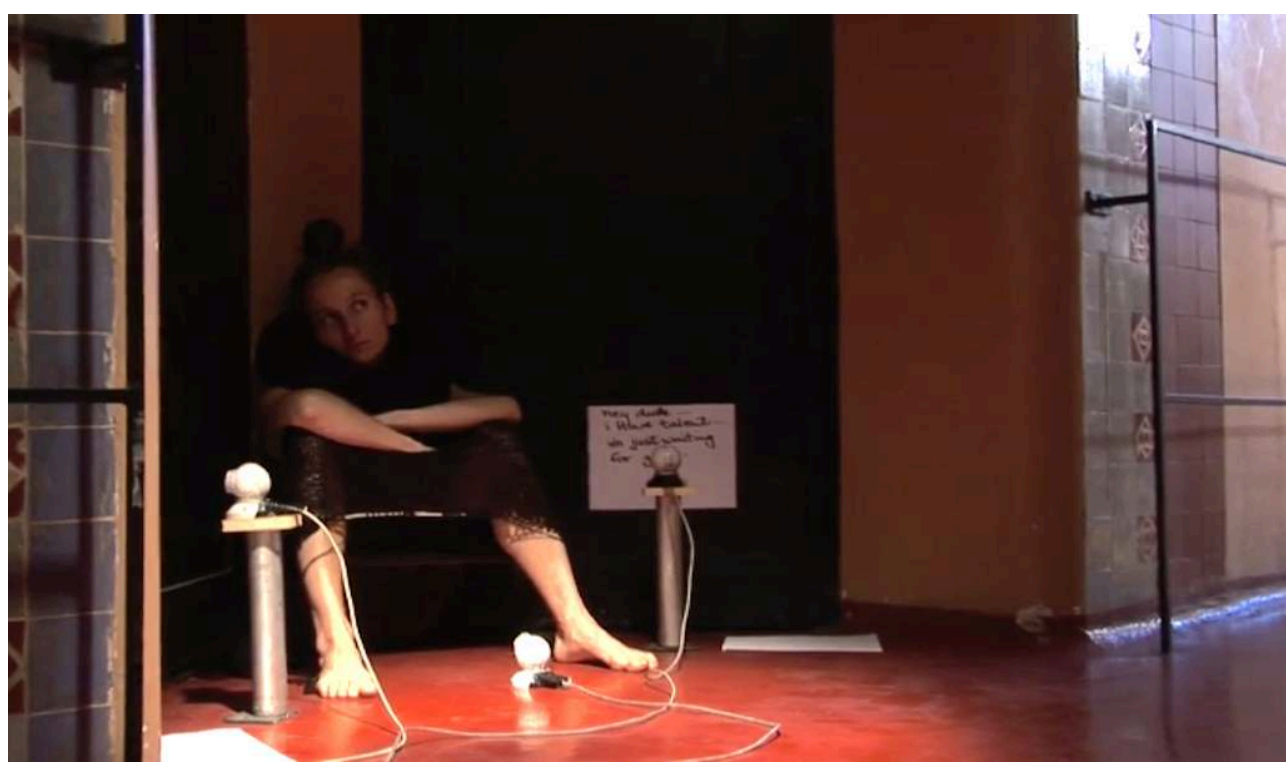

Photogramme de la captation réalisée par Philippe Lainé

(c) Sweety Pie, 2008

Il arrive parfois qu'en l'absence de l'auteur quel qu'il soit - Robyn, César, Tartempion ou Dieu, autrement dit le créateur minuscule ou majuscule (ou bien le directeur du théâtre) -, Saddam Hussein, George W. Bush, Vladimir Poutine, Nicolas Sarkozy ou Louis XIV fasse irruption. Il arrive aussi que l'acteur-danseur abandonné, seul ou avec quelques commères'pères, trouve un recours plus efficace auprès d'un dieu des machines, Thabo (Pule), Nico (Moremi) ou Maxime (Rebière), en général off stage mais présent, lui, dans les coulisses ou en régie, et susceptible de répondre à l'injonction ou à la supplication. Efficacité littérale dans la mesure où, si l'appel au secours produit un effet (la lumière s'allume ou la musique s'arrête, par exemple), il ne permettra pas pour autant de sauver les meubles ni d'échapper au désastre. Dans tous les cas, on l'aura compris, tout en ouvrant subrepticement le débat sur la place de l'auteur, ce que Robyn Orlin fait briller par l'absence de celui-ci, c'est l'autorité au sens générique, la détention du pouvoir. Ou, pour le dire autrement (ceci étant la conséquence de cela), avec ce motif dramaturgique décliné de pièce en pièce, elle file la métaphore du pouvoir - avec ses figures omniprésentes, mais en surplomb, hors d'atteinte - comme centre vide.

Cependant, dans le raté de l'auteur (qui traduit aussi son humanité), dans la vacance du pouvoir qu'il révèle, on pourrait entrevoir une opportunité. Une suggestion, une invitation, une incitation, aussi ténue soit-elle, aussi risquée soit-elle, à tirer parti de la situation pour (essayer d') ébranler le système. Après tout, Robyn Orlin n'a pas attendu la fin de l'apartheid pour se lever contre l'oppression à tous les étages, et soutenir les vertus de la démocratie. Mais, indépendamment des périls inhérents à toute prise de pouvoir $^{19}$, le rater est contagieux et il n'est pas l'apanage de l'auteur.

\section{L'acteur-danseur, au risque de l'auteur}

Qu'il décide ou non de profiter de son absence - absence qui pourrait être un leurre pour prendre sa place ${ }^{20}$, dans tous les cas, sa sujétion expose l'interprète aux ratages de 
l'auteur. Dans le grand théâtre d'Oklahoma, « qui emploie tout le monde et met chacun à sa place ${ }^{21} "$, acteurs et machinistes sont toujours en première ligne.

C'est à nouveau, quoi qu'en présence, un rendez-vous raté qui forme l'arrière-plan dramatique de Hey Dude... Rendez-vous raté en ce sens qu'entre la danseuse et la chorégraphe, entre (le personnage) Vera Mantero et (le personnage) Robyn Orlin, la rencontre n'a pas eu lieu, elle s'est traduite par un complet malentendu, l'impossibilité d'un dialogue. Et quand la pièce commence (ou recommence, puisqu'une séquence filmée et retransmise en direct précède l'entrée en scène proprement dite, laquelle se fait par la salle), on apprend que, de pièce, il n'y a/aura pas. Et pas seulement pour les motifs exposés en préambule - à savoir que, Robyn ne sachant pas tricoter, le costume de Vera n'est pas prêt. Mais parce que, de ces longues heures de travail, de répétition, de tentative, d'explication, accumulées par Vera et Robyn jusqu'à cet instant $\mathrm{T}$, rien n'est sorti. Voilà l'argument qui nous est servi et qui, Vera le rappelle incidemment, n'a strictement rien à voir avec l'horizon d'attente que déploie la note d'intention du spectacle - où il serait question des sans-abri et de leurs stratégies de survie.

Il faut toujours se méfier des apparences, pourtant le documentaire ${ }^{22}$ réalisé en 2005, dans les coulisses de la création de Hey Dude..., offre à la pièce un intéressant prolongement. En voici la transcription de deux extraits.

Robyn Orlin: «Ce qui m'intéresse actuellement dans mon travail, c'est ce qui se passe au cours du processus [...]. Je me concentre sur ce que je peux obtenir de la part du performer et c'est à partir de là que je commence à bâtir la pièce. »

Vera Mantero : « À un moment donné, j'ai dit à Robyn : “Je ne comprends pas où on veut aller, je ne me sens pas bien dans ce qu'on est en train de faire [...]." Et elle me disait : "C'est ça que doit être le spectacle, ça doit être sur ça." [...] Dès le début, il y avait [chez elle] cette envie qu'il y ait pour moi une difficulté de jouer, que je ne puisse pas jouer. »

Admettons que le rater soit le biotope de l'artiste Robyn Orlin. Faut-il en déduire pour autant que celle-ci puise sa substance dans les fragilités, sinon dans les échecs (réels ou supposés, effectifs ou fictifs) de ses interprètes ? Ce n'est pas sûr. Pourtant, le thème du performer mis en difficulté ou pris en défaut est également récurrent chez elle. 
Julia Burnham dans Beauty remained for just a moment..., Robyn Orlin, 2012

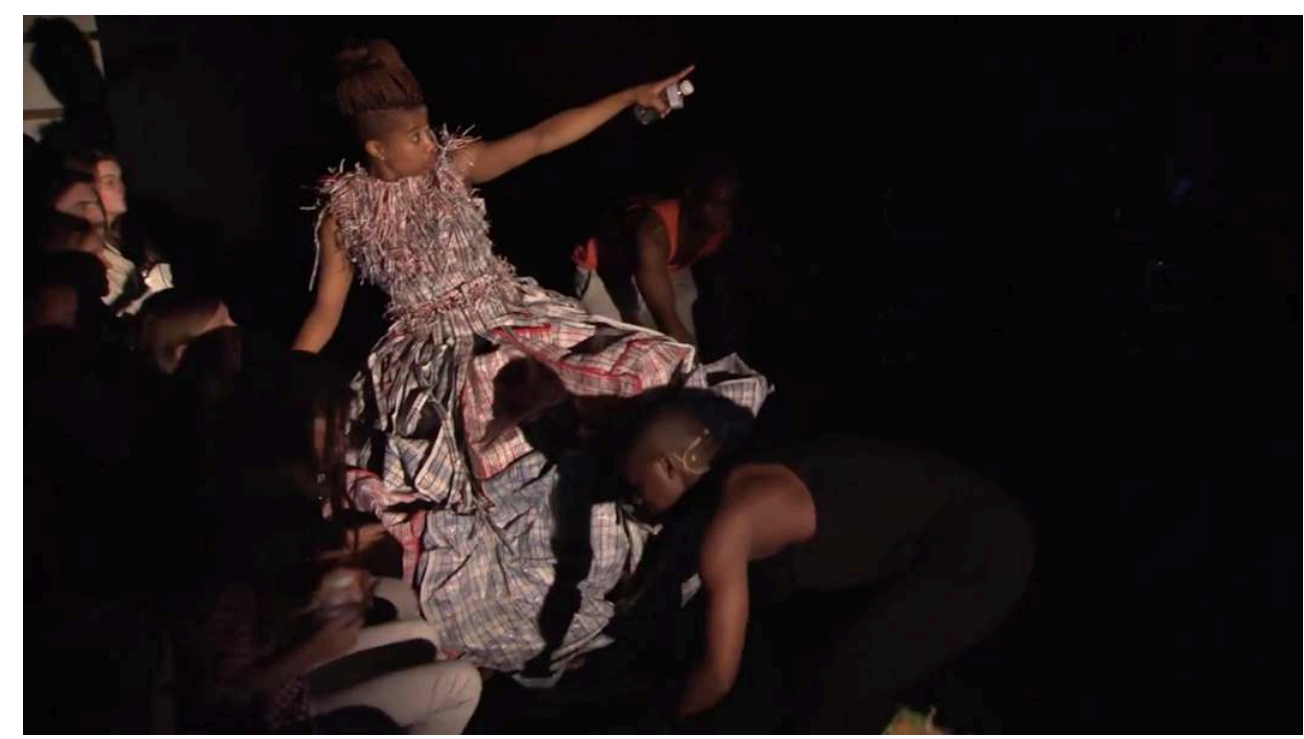

Photogramme de la captation du spectacle, réalisée par Luc Riolon, filmée à La Ferme du BuissonScène Nationale de Marne-la-Vallée

(C) arte France, 2012

Ainsi, dans le solo (accompagné) Call it... kissed by the sun... better still the revenge of geography... (Appelons-le... embrassé par le soleil... ou, mieux encore, la revanche de la géographie, 2010), Ibrahim Sissoko rate son entrée, il pulvérise un pan du décor dont il révèle ainsi l'équilibre précaire et se confond en excuses. Dans In a world full of butterflies, it takes balls to be a caterpillar... some thoughts on falling... (Dans un monde empli de papillons, il faut des couilles pour être une chenille... quelques pensées sur la chute, 2013), l'une des répliques d'Elisabeth Bakambamba Tambwe dit à peu près ceci : "Robyn m'a demandé si je ne pouvais pas me casser la gueule avec un peu plus d'élégance.» Dans Beauty remained for just a moment..., Julia Burnham se demande en aparté s'il était bien nécessaire de faire six ans d'études pour en arriver là, se retrouver à jouer ce rôle dans cette pièce. Dans Although I live inside... my hair will always reach towards the sun... (Bien que je vive à l'intérieur... mes cheveux seront toujours offerts au soleil, 2004), Sophiatou Kossoko prend le public à témoin des basses tâches auxquelles elle, qui est une "danseuse contemporaine " tout de même, est contrainte par la chorégraphe. Et, dans ... have you hugged, kissed and respected your brown Venus today? (... avez-vous étreint, embrassé et respecté votre Vénus marron aujourd'hui ?, 2011), Dudu Yende, « qui est une actrice, elle », explose, récrimine et s'épanche, dénonçant, dans le désordre, les maltraitances que Robyn lui fait subir, la vie de chien qu'elle lui fait mener et la médiocrité dans laquelle elle entraîne ses interprètes ${ }^{23}$. 
Dudu Yende dans ... have you hugged, kissed and respected your brown Venus today?, Robyn Orlin, 2011

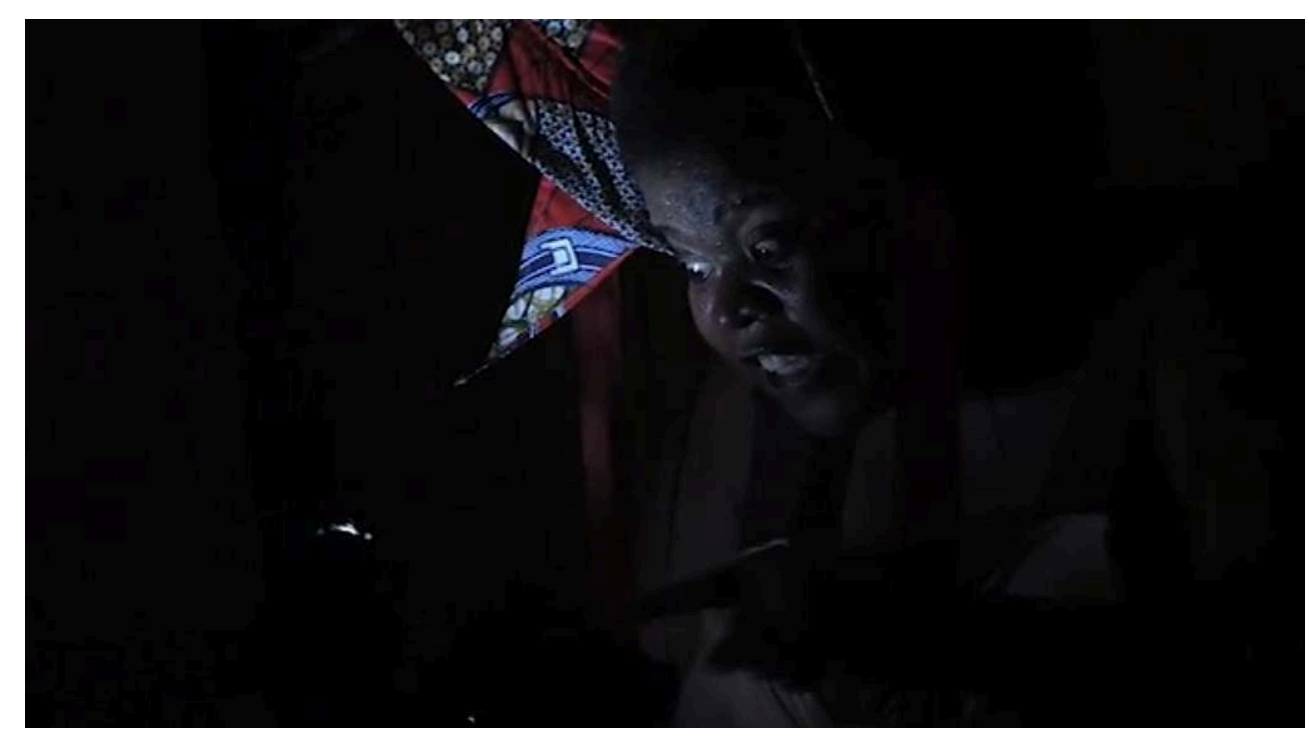

Photogramme de la captation du spectacle filmée par Corinne Dardé et Raymond Macherel à la Maison de la Musique et de la Danse, Nanterre, le 6 décembre 2011

Il ne faut pas se fier aux apparences de la fiction, cependant le témoignage de Vera Mantero - «Dès le début, il y avait cette envie qu'il y ait pour moi une difficulté de jouer, que je ne puisse pas jouer »- nous donne une indication sur la manière dont Robyn Orlin conduit le processus de ses créations scéniques et expose, au-delà de leur exposition générique, ses collaborateurs au rater. Il existe, en effet, des témoignages convergents.

Celui de Sophiatou Kossoko pour Although I live inside..., par exemple :

J'ai vraiment eu l'impression de vivre la création de plusieurs spectacles en même temps. À deux jours de la première, nous n'avions pas encore réalisé un seul filage ! Elle avait déjà en tête un canevas très précis mais elle le gardait pour elle, et je restais dans l'inconnu. Il y a une réelle prise de risque dans sa manière de travailler. Cela exige une complète disponibilité et une grande ouverture de la part de l'interprète ${ }^{24}$.

27 Ou celui du réalisateur Philippe Lainé qui a travaillé sur une dizaine de créations de Robyn Orlin :

Elle lance des pistes, souvent larges, et vous demande à partir de cela de lui donner de la matière dans laquelle elle puisera ce qui l'intéresse. Cela laisse une énorme liberté, qui peut être dangereuse si l'on n'a pas l'habitude de travailler avec autant de marge de manœuvre. On peut s'y perdre $[. . .]^{25}$. 
Marie-Agnès Gillot, répétitions de L'Allegro, il Penseroso ed il Moderato, Robyn Orlin, Opéra de Paris, 2007

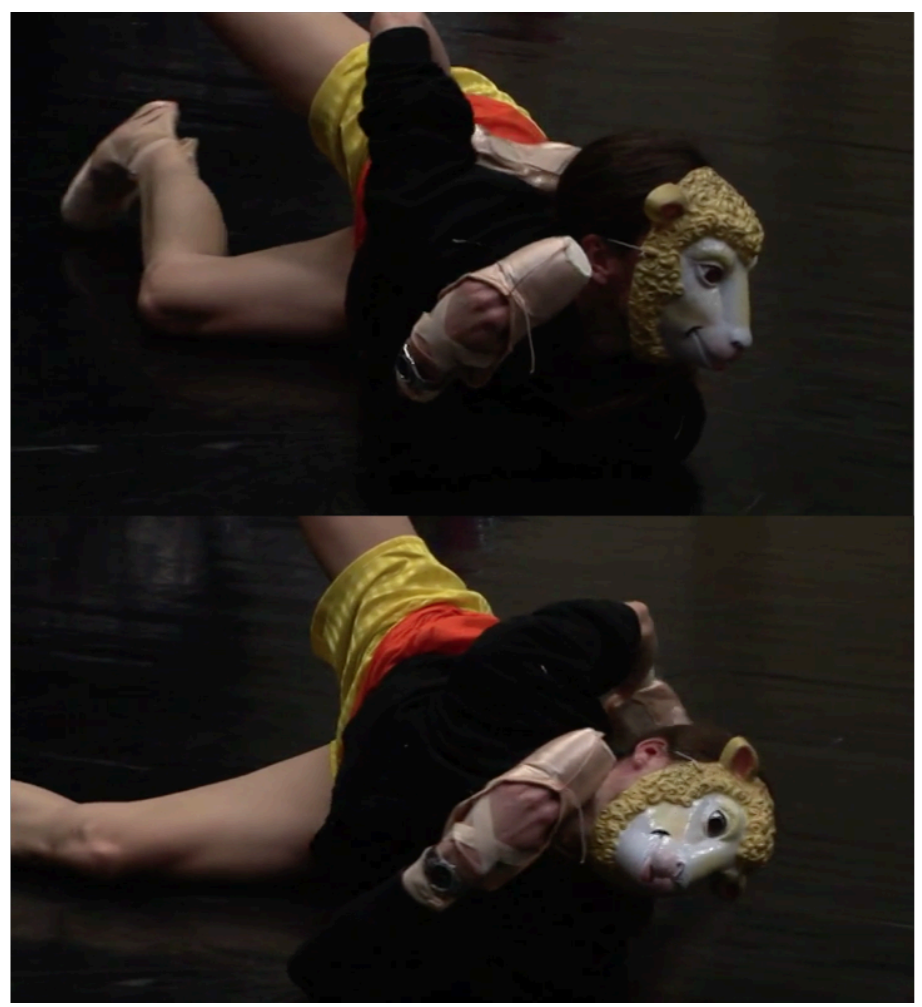

Photogrammes de Robyn Orlin, de Johannesburg au Palais Garnier, réal. Philippe Lainé et Stéphanie Magnant

(c) Zadig, Ina, arte France, 2008

Il n'y a pas de violence dans les relations que Robyn Orlin instaure avec ses partenaires pendant le processus de création. Au contraire. Même s'il est vrai qu'elle est parfois en retard et que sa méthode (son absence de méthode apparente) est assez déroutante, elle fait tout son possible pour donner une place à chacun, elle se montre pleine d'attention et curieuse de tous, au-delà du cadre strict du travail, rassure et sollicite ( $I$ I need your help!", «I am with you ", «j'ai besoin de vos lumières ", " ce que vous avez à me dire, chacun d'entre vous, est très important»), incite les acteurs-danseurs-chanteurs à " se lâcher ", à faire toute sorte de tentatives ("it's just an experiment »), à s'approprier les situations ("make your own version...»), et aussi à jouer, à s'amuser («just play, people! have fun!»).

Pourtant, si l'on se réfère au film de Philippe Lainé et Stéphanie Magnant, Robyn Orlin, de Johannesburg au Palais Garnier, le "risque de se perdre" est revenu comme un leitmotiv dans la collaboration de l'artiste avec les danseurs du corps de ballet de l'Opéra de Paris pour la création, en 2007, de L'Allegro, il Penseroso ed il Moderato de Haendel. À titre d'exemple, on citera cette séquence dans laquelle le danseur étoile Nicolas Le Riche, commentant la phrase qu'il vient de danser, déclare, à la grande satisfaction de Robyn Orlin : "Ça n'a l'air de rien, mais c'est difficile !» Ou cette autre, dans laquelle Alice Renavand, travaillant sous la direction de l'assistant-maître de ballet Fabrice Bourgeois, s'entend dire : «C'est bien intéressant quand c'est coincé [...]. Il ne faut pas que ce soit facile, Robyn va dire "too easy", sinon!» Cette autre, enfin, 
dans laquelle Yann Bridard, répétant seul avec Robyn Orlin, concède qu'il est " perdu », qu'il est en train « de [s] fermer », qu'il se " sent seul ».

Même dans un cadre aussi contraint et hiérarchisé que celui de l'Opéra de Paris, Robyn Orlin se refuse à diriger de façon classique : «Tant que tu n'attends pas que je te dise quoi faire, ça va ${ }^{26}$ ", dit-elle à un danseur. Quant à la difficulté à laquelle elle confronte ses interprètes, elle en convient elle-même. Voici, toujours sur les répétitions de L'Allegro, quelques-unes de ses indications à Marie-Agnès Gillot :

Ce que tu es en train de créer, c'est ton propre vocabulaire. Dès que tu rentres en terrain connu, que tu utilises un vocabulaire familier, ça perd de sa force. [...] Il n'y a rien de plus difficile que de créer son propre vocabulaire. [...]

J'aime la lutte et c'est ça qui m'intéresse. C'est intéressant de montrer la lutte et de voir le mouvement qu'elle produit ${ }^{27}$.

\section{Et le spectateur, dans tout ça? Épinglé dans un coin du miroir}

31 Sachant l'engagement actif de Robyn Orlin contre toute forme de discrimination, on ne s'étonnera pas que le spectateur de son théâtre ne soit, dans son traitement, nullement discriminé. Ce qui, dans le contexte qui nous occupe, signifie que, tout comme travailler avec elle, aller voir une pièce de Robyn Orlin, c'est aussi, déjà, s'exposer au rater. À partager un rendez-vous raté avec elle, à assister aux ratages et ratés de l'un ou l'autre de ses performers ou techniciens, ou à faire soi-même l'expérience du rater.

C'est à nouveau la question de la place et de ses incidences sur le regard qui est en jeu ici. C'est le fait qu'il n'y ait, en définitive, ni bon ni mauvais côté, pas de qualification ni de hiérarchisation des espaces, pas d'assignation des rôles, pas de perspective unique ni dominante. C'est la plasticité, la réversibilité ou l'absence des catégories et des frontières. C'est, symétriquement, le règne de l'indistinction, de la désorientation, du brouillage, du mixage. De l'incertitude.

Pour le spectateur de Robyn Orlin, le risque du rater tient surtout à la pratique, fréquente sinon systématique chez elle, des interactions avec le public. Ici encore, les modalités de cette pratique sont diverses dans leur forme, leur nature comme dans leur amplitude. Il peut s'agir de céder sa place à un interprète ou de l'accueillir sur ses genoux, de lui confier son pull, sa chemise, une chaussure, son soutien-gorge ou un chewing-gum longuement mâché, de lui (faire) passer un objet (un sac, une cigarette, une boisson), de tenir un œillet entre ses dents pour contribuer à la construction d'une image ou de participer au déroulement-déploiement d'un voile depuis la scène jusqu'aux travées supérieures de la salle... L'action à laquelle est convié le public peut être collective : lancer d'avions en papier, de bouteilles d'eau en plastique, gargarismes sonores sur trois tons, déclenchement de l'alarme de son téléphone portable ou ola. Elle peut répondre à une sollicitation directe (et parfois directive : «Madame, repeat after me ", parfois plaintive : " help me ») ou dépendre de votre bon vouloir, par exemple dans Daddy... ou dans Although I live inside..., lorsque tout ou partie du public est engagé à rejoindre le-la ou les acteurs-danseurs pour acter-danser avec elle ou lui ou eux. Elle peut vous laisser dans votre fauteuil ou vous obliger à le quitter (puisque, rappelons-le, la question de la place est en jeu). Elle peut vous rassembler dans la communion et le partage (d'une danse, d'une image, d'une pétition à signer, d'une banane, d'une « part 
du gâteau » ou d'une orange). Elle peut aussi vous isoler dans la foule... par exemple en vous forçant (avec douceur) ou en vous invitant (avec rudesse) à monter sur scène.

Dans tous les cas, et c'est en cela qu'il est permis, me semble-t-il, d'assimiler ces interactions à des ratés, le spectateur, seul ou en groupe, se voit contraint à sortir de sa position, à prendre part au jeu et à s'exposer ainsi à la fois au rater et aux regards de ses pairs. Or, qu'il s'y prête ou non, dans tous les cas, c'est "raté, raté et encore raté ». "Celui qui critique ou repousse le jeu, est déjà entré dans le jeu ${ }^{28}$ ", écrit Maurice Blanchot.

La mise en œuvre de telles interactions soumet le destinataire à cette expérience que Marie José Mondzain, à propos de La Colonie pénitentiaire de Kafka, désigne comme «l'énigme des basculements»:

La question de l'assistance [...] désigne la place du spectateur assistant à un spectacle durant lequel il peut ne rien lui arriver; mais l'assistance est aussi le terme qui désigne la présence secourable que l'on est en droit d'attendre d'un spectateur et d'un témoin auquel il arrive quelque chose quand il en est spectateur. Il s'agit donc de sa capacité potentielle d'être acteur et non spectateur indifférent et épargné. [...]

Le voyageur ne sort donc pas indemne de sa visite. Sa hâte de partir et son malaise trahissent son angoisse justifiée, celle d'être finalement une pièce involontaire et affolée du système auquel il ne peut que feindre d'être étranger ${ }^{29}$.

Même si Robyn Orlin n'est pas naïve quant au pouvoir de l'art, c'est bien de cela qu'il s'agit pour elle. De la conviction que le théâtre n'est pas un lieu séparé - du monde ou de la société. Une conviction qui prend forme également, lorsque l'action dramatique se déploie à l'extérieur de l'espace scénique - dans Hey Dude... -, lorsqu'elle se poursuit à l'extérieur du théâtre - dans f... (untitled) ou, plus radicalement encore, dans The future may be bright, but it's not necessarily orange... !!! (L'avenir peut être lumineux, mais il n'est pas forcément orange...!!!, 2000)-, ou lorsque, par le biais de la vidéo, l'extérieur est « importé » à l'intérieur, comme c'est le cas dans L'Allegro, par exemple.

Je pense qu'il est très important pour un public de théâtre d'être pris à parti, d'être interpelé [...], d'être poussé à agir, à être actif. [...] Traditionnellement, le spectateur est assis là, et il regarde. Mais, selon moi, il faut l'engager dans le spectacle [...] $]^{30}$.

Pour « engager » le spectateur dans le spectacle, Robyn Orlin, on l'a vu, n'est pas à court d'idées. Et, parmi d'autres, elle joue précisément sur la nécessité d'ajuster son regard ou sur la dissémination des points de vue. L'usage de la vidéo est, en la matière, un allié de choix.

Dans Daddy... par exemple, quatre moniteurs, reliés à plusieurs caméras, sont suspendus aux quatre coins du plateau et diffusent tout au long du spectacle des images captées en direct, pour l'essentiel dans les coulisses du spectacle. Images parasites qui sollicitent l'attention et perturbent la vision du spectacle tout en en montrant le revers, ces images en noir et blanc, de qualité médiocre, évoquent celles qui sont produites par les caméras de surveillance (et voilà la question du pouvoir qui revient, sous les traits de Big Brother). Cependant, dans le cours de la pièce, l'un des acteurs-danseurs (Gerard Bester, encore, dans le personnage duquel on pourrait d'ailleurs reconnaître l'instrument du pouvoir), présentant la séquence dansée qui va suivre, "une chorégraphie de Robyn Orlin intitulée "Les roses" ", prévient qu'il s'agit d'une danse pour la caméra, qu'il faut donc la regarder non en direct, sur la scène, mais via les moniteurs qui l'encadrent et qui, effectivement, en retransmettent en direct l'image en plongée. Ce qui place le spectateur devant un dilemme et génère chez lui un sentiment 
de frustration: deux images différentes et simultanées de la danse lui sont ainsi proposées, l'une directe, l'autre médiatisée, qu'il ne peut saisir en même temps puisqu'elles sont situées dans deux champs de vision séparés.

Daddy..., Robyn Orlin, 1999

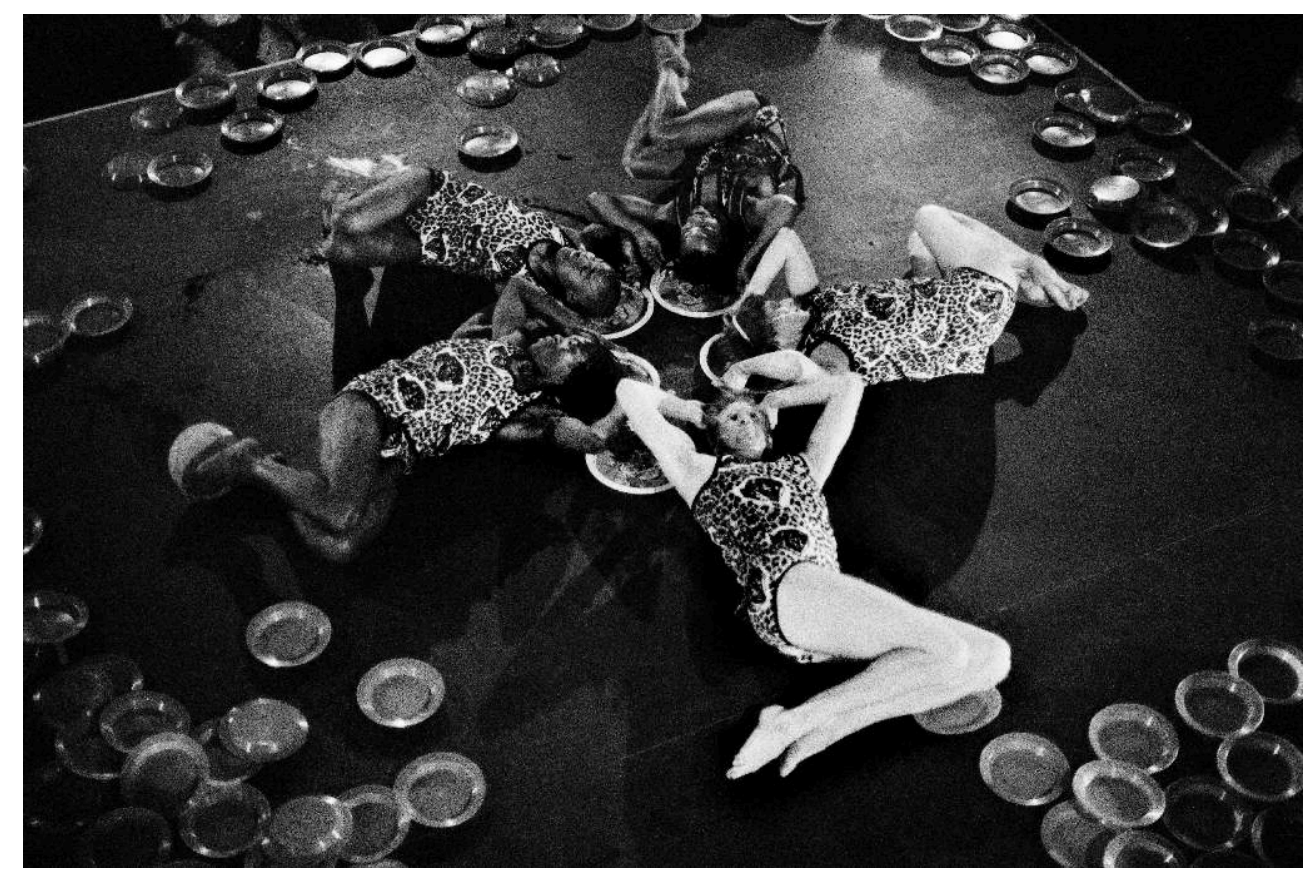

(c) John Hogg

Un principe analogue de vision diffractée et de perspective démultipliée est mis en jeu dans L'Allegro, en recourant à une technologie beaucoup plus sophistiquée (contexte oblige ? ou permet ?). Le dispositif éclaté imaginé par Robyn Orlin prévoit en effet trois aires de « jeu » disjointes, mais interdépendantes : la fosse d'orchestre et les premiers rangs où les choristes sont installés parmi les spectateurs; le plateau nu, saturé de lumière, sur lequel évoluent les danseurs et les chanteurs solistes ; et, en surplomb, un écran panoramique qui occupe toute la moitié supérieure du cadre de scène, sur lequel, pendant toute la durée du spectacle, est projetée une vidéo où des images des interprètes présents sur le plateau viennent s'incruster en temps réel. Là encore, le spectateur se voit contraint à effectuer des choix... et à rater du même coup une partie du spectacle. 
L'Allegro, il Penseroso ed il Moderato, Robyn Orlin, Opéra de Paris, 2007

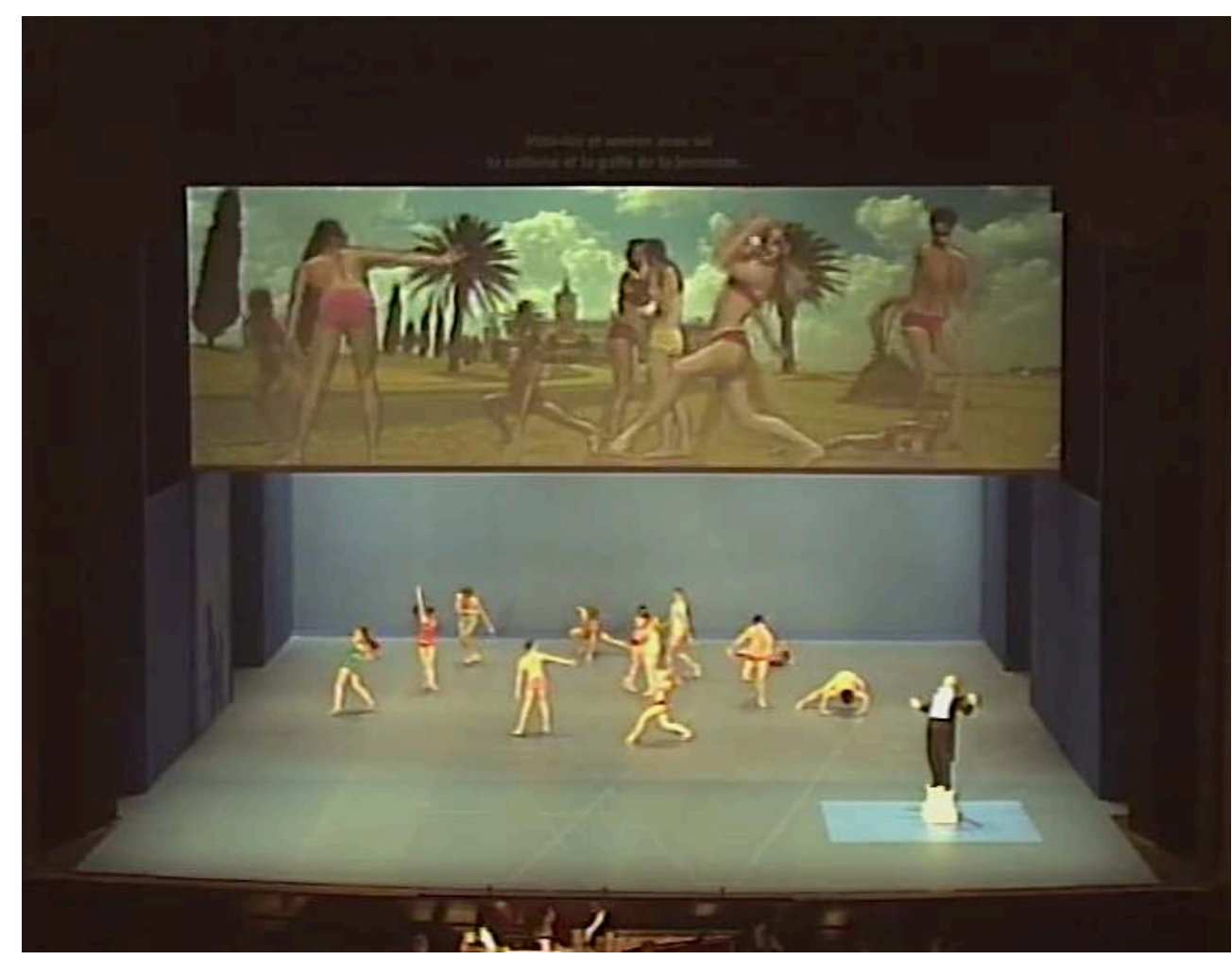

Photogramme d'une captation de service

40 L'utilisation de la vidéo permet donc de remplacer ( $f .$. (untitled) et Hey Dude) ou de prolonger la présence des acteurs-danseurs-chanteurs par leur image filmée (plans larges ou gros plans sur un détail de leur anatomie). Elle permet aussi de tendre un miroir au public, de lui offrir un « reflet » collectif ou de focaliser l'attention sur un ou quelques-uns des spectateurs, comme c'est le cas dans Hey Dude... ou dans ... have you hugged, kissed and respected your brown Venus today? - spectateur(s) qui se trouve(nt) ainsi projeté(s) sur scène, sans même avoir à quitter sa/leur place.

41 Elle peut enfin être associée à la stratégie qui consiste à convoquer physiquement des spectateurs sur scène, comme dans And so you see... our honourable blue sky and ever enduring sun... can only be consumed slice by slice... (Et comme vous le voyez... notre honorable ciel bleu et notre soleil éternel... ne peuvent être consommés que tranche par tranche..., 2016), une pièce qui conjugue les possibles, multiplie les tableaux et met du miroir dans le miroir.

Le premier raté auquel est d'emblée confrontée l'assistance ressemble à une transgression, légère : dans un dispositif classique, frontal, l'interprète soliste, Albert Silindokuhle Ibokwe Khoza, est placé dos au public et le restera pendant la majeure partie de la pièce. Des caméras, toutefois, semblent venir au secours des spectateurs l'une en particulier, étant placée presque au centre du plateau, au lointain, et manipulée par un opérateur. Elles captent en temps réel mais pas en continu, en plan large ou moyen ou gros, la face (ou les pieds) d'Albert, tandis que les images ainsi produites sont retransmises sur l'écran déployé en fond de scène, derrière l'homme à la caméra. Cette fois, l'acteur et son image, projetée à l'arrière-plan, peuvent certes être saisis simultanément par le regard, mais un constant travail d'accommodation s'impose: en effet, indépendamment de la différence d'échelle entre Albert et sa 
représentation en vidéo, les images projetées ne proviennent pas toutes de la même source. De plus, le cadre et la profondeur de champ de la caméra placée au lointain et « opérée » en direct par Thabo Pule varient. La silhouette d'Albert (grandeur) nature se détache donc parfois sur l'image en gros plan de son visage (ou de ses pieds), mais il arrive aussi que le spectateur se surprenne lui-même à l'image, à regarder/regardant Albert et/ou son image. Enfin, dans une séquence qu'on pourrait intituler: "The Nubian Queen » (tel en est du moins l'argument), Albert Ibokwe Khoza utilise un miroir, dont le reflet est, lui aussi, diversement capté par la caméra. Ce qui produit de somptueux effets kaléidoscopiques, tout en générant un risque de rater majeur, c'est-àdire de surexposition, pour l'heureux élu, seul dans la foule, qu'Albert épinglera dans un coin de son miroir $^{31} \ldots$

Albert Ibokwe Khoza dans And so you see.., Robyn Orlin, 2016

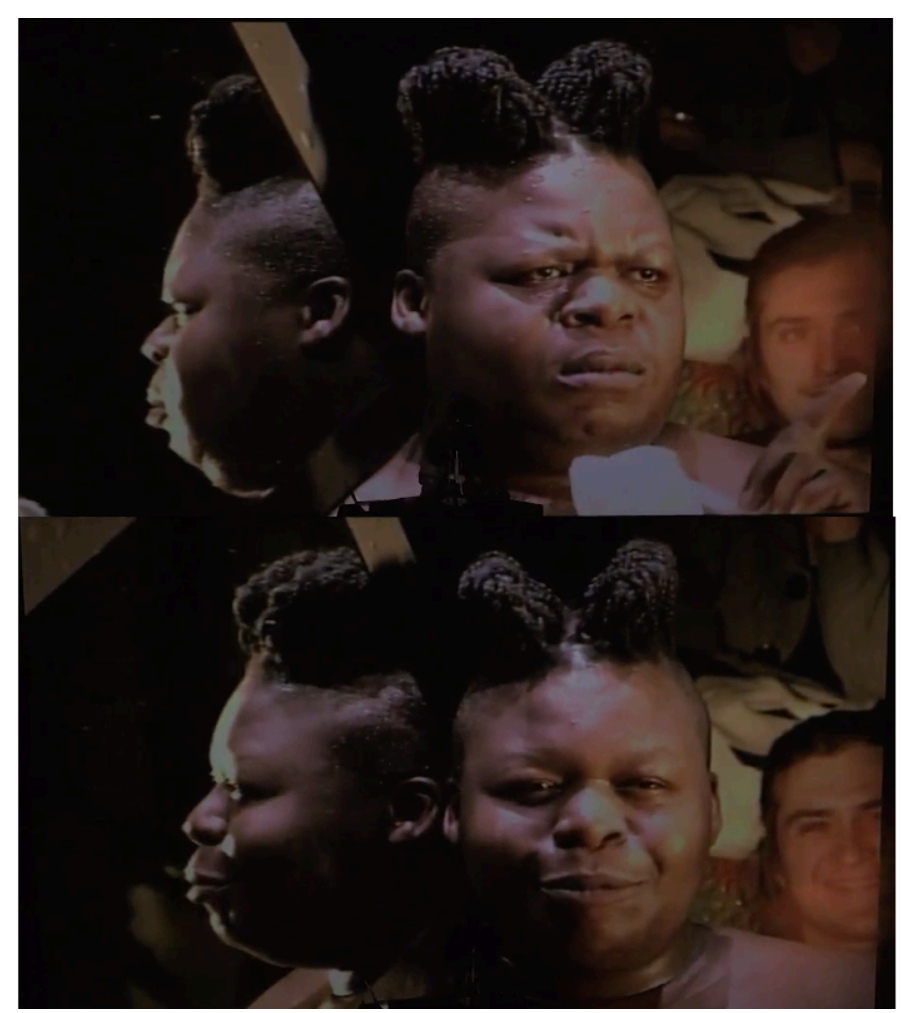

Photogrammes de la captation du spectacle, réalisée par Éric Legay 


\section{Pour « rater mieux » enfin, sans en dire les vertus}

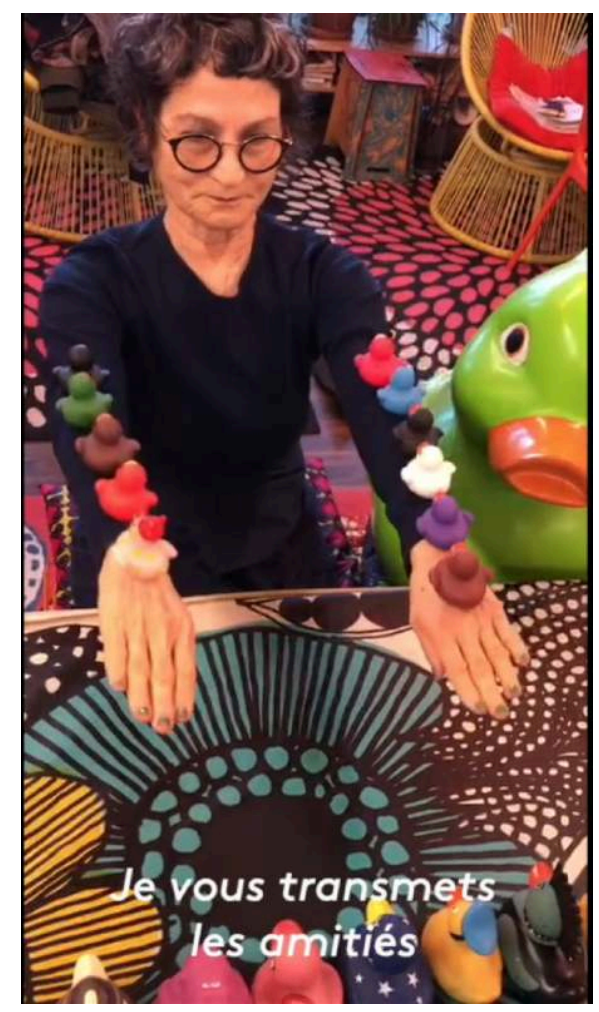

Photogramme de Robyn Orlin\#jedansealamaison, mars 2020

Commande de Chaillot-Théâtre National de la danse (https://vimeo.com/412365586)

Pour Robyn Orlin, l'espèce humaine est diverse, multiple et, en dépit de ses singularités, elle est indivisible, non-hiérarchisable. Ses représentants devraient donc être égaux en droits, et égaux, du même coup, dans l'expérience du rater. Cet horizon, cette utopie, elle s'efforce, on le voit, de l'actualiser à tous les étages dans son théâtre. Un théâtre qui propose un reflet du monde et où, comme dans la vraie vie, règne le chaos.

Dans un repli du «rater mieux » selon Robyn Orlin, on pourrait enfin s'attacher à l'attention particulière que manifeste son écriture pour le tiers- et le quart-monde, pour les agents d'entretien, les gardiens de musée, les habitants des townships et les sans-abri, pour les ouvriers en général et les mineurs en particulier, pour les marchands ambulants ou à la sauvette, pour les derniers de la classe ou les petits délinquants et, de manière plus générale, pour les invisibles, les sans-nom, les exclus de la société et les oubliés de l'histoire...

Alors, pour conclure sur le rater avec une image de Robyn Orlin entourée d'une armada de canards en plastique ${ }^{32}$, une histoire encore, qui ne lui est pas propre, mais qui éclaire, me semble-t-il, la problématique du « rater mieux, selon Robyn Orlin ».

Il s'agit forcément de l'histoire d'un raté - figuré non par un canard, mais par un petit oiseau tombé du nid-, qui, comme celle du monde et du pantalon, connaît de nombreuses variantes : c'est un tout petit oiseau à peine sorti de sa coquille et qui ne sait pas encore voler. Voilà qu'il tombe du nid familial, il est seul, il a froid et il piaille de désespoir. Quelqu'un ou quelqu'une, jeune ou vieux, pauvre ou riche, malade ou en bonne santé, vient à passer. Sensible à la souffrance de l'oiseau, ileelle le ramasse et, 
avisant à proximité une bouse de vache encore fumante, l'y dépose. L'oisillon est alors tout heureux, il a chaud, il se sent bien et il se met à chanter. Passe un renard qui, entendant le chant de l'oiseau, fonce droit sur lui et n'en fait qu'une bouchée... Il y a trois morales à cette histoire, mais pour la rater mieux, nous ne les exposerons pas ici ${ }^{33}$.

\section{BIBLIOGRAPHIE}

\section{Biblio-filmographie}

ARENDT Hannah, La liberté d'être libre. Les conditions et la signification d'une révolution, trad. Françoise Bouillot, Payot, 2020.

BECKETT Samuel, Le Monde et le Pantalon, suivi de Peintres de l'empêchement, Minuit, 1990.

BLANCHOT Maurice, L'Écriture du désastre, Gallimard, « nrf », 1980.

BOURDIÉ Annie, « Art chorégraphique contemporain d'Afrique, enjeux d'une reconnaissance », Marges [En ligne], 16 | 2013, mis en ligne le 15 mars 2014. URL : http://journals.openedition.org/ marges/263 ; DOI : https://doi.org/10.4000/marges.263 [consulté le 15 août 2020].

DIDI-HUBERMAN Georges, Ce que nous voyons, ce qui nous regarde, Minuit, 1992.

HESPEL Olivier, Robyn Orlin, fantaisiste rebelle, Éditions de l'Attribut / CND, 2007.

KAFKA Franz, L’Amérique, trad. Alexandre Vialatte, Paris, Gallimard, « folio », 1973.

LAINÉ Philippe (réal.), Hey Dude... - captation @ Sweety Pie, 2008.

LAINÉ Philippe (réal.), Who is Robyn Orlin ?, 4'30, Sweety Pie Prod., 2011.

LAINÉ Philippe, MAGNANT Stéphanie (réal.), Robyn Orlin, de Johannesburg au Palais Garnier, 87', Zadig Productions, Arte France, INA, 2008.

MAGNANT Stéphanie, LAINE Philippe (réal.), Hey Dude..., 13'30, Sweety Pie, 2005.

MONDZAIN Marie José, K comme Kolonie. Kafka et la décolonisation de l'imaginaire, La fabrique éd., 2020.

ORLIN Robyn, RICHEUX Marie, « Par les temps qui courent », France Culture, 6/11/2019. URL : https://www.franceculture.fr/emissions/par-les-temps-qui-courent/robyn-orlin

PALAZZOLO Claudia, Mise en scène de la danse aux Expositions de Paris, 1889-1937. Une fabrique du regard, L'œil d'or, 2017.

SCHÜTZ Alfred, L'Étranger. Un essai de psychologie sociale (1944), suivi de L'homme qui rentre au pays (1945), trad. de l'anglais Bruce Bégout, Allia, 2010.

\section{Corpus d'œuvres de Robyn Orlin (sélection)}

And so you see... our honourable blue sky and ever enduring sun... can only be consumed slice by slice..., un solo de Robyn Orlin pour Albert Ibokwe Khoza, création au Festival Montpellier Danse, juin 2016. 
Beauty remained for just a moment then returned gently to her starting position ... une pièce de Robyn Orlin, avec Julie Burnham, Oscar Buthelezi, Teboho Letele, Sunnyboy Motau, Otto Nhlapo, Muzi Shili, Thandi Tshabalala, création à la Biennale de Danse de Lyon, septembre 2012.

daddy, I've seen this piece six times before and I still don't know why they're hurting each other... A piece for six dancers and a stage, de Robyn Orlin, avec Gerard Bester, Nico Moremi, Toni Morkel, Pule Molebat, Nelisiwe Xaba, Dudu Yende, commande du FNB Dance Umbrella, Johannesburg, mars 1999.

... have you hugged, kissed and respected your brown Venus today? une pièce de Robyn Orlin, avec Ann Masina, Dorothée Munyaneza, Angela Simpson, Elisabeth Bakambamba Tambwe, Dudu Yende, création au Grand Théâtre du Luxembourg, novembre 2011.

Hey dude... I have talent... I'm just waiting for God..., un solo de Robyn Orlin, pour et avec Vera Mantero, commande du Festival Danse à Aix, création au 3bis F, Aix-en-Provence, juillet 2005.

L'Allegro, il Penseroso ed il Moderato, de G. F. Haendel, livret de Charles Jennens d'après John Milton, chorégraphie et vidéo Robyn Orlin, direction musicale William Christie, avec Nicolas Le Riche, Yann Bridard, Alica Renavand et le Ballet de l'Opéra de Paris, Kate Royal, Toby Spence, Roderick Williams, Daniel Krähmer, l'Orchestre et le Chœur des Arts florissants, commande de l'Opéra National de Paris-Palais Garnier, avril 2007.

\section{NOTES}

1. Cf. Vera Mantero, Une mystérieuse chose, a dit e.e. Cummings*, création pour Culturgest, en hommage à Joséphine Baker, Lisbonne, 1996.

2. Pour l'essentiel, la documentation relative à Robyn Orlin relève, soit de la communication (brochures, feuilles de salle), soit de la presse (information, réception critique). Depuis 1999, la plupart de ses pièces et performances ont fait l'objet de captations dont certaines sont consultables sur le site numeridanse (www.numeridanse.tv). Outre ces sources, conséquentes mais disséminées, il existe deux monographies: Olivier Hespel, Robyn Orlin, fantaisiste rebelle, Éditions de l'Attribut / CND, 2007 ; et Robyn Orlin, de Johannesburg au Palais Garnier, réalisation Philippe Lainé et Stéphanie Magnant (87', Zadig Productions, Arte France, INA, 2008).

3. Samuel Beckett, Le Monde et le Pantalon, suivi de Peintres de l'empêchement, Minuit, 1990 , p. 9.

4. Qui, comme je viens de le vérifier (conversation téléphonique avec Philippe Lainé, Tourtour-Paris, 12 août 2020), ne connait ni Gertrude Stein ni Alice Toklas.

5. Who is Robyn Orlin ?, réalisation Philippe Lainé (4'30, Sweety Pie Prod., 2011).

6. «Je ne sais pas qui je suis... je ne sais pas qui je suis. Je ne sais pas où je vis. Je ne sais pas si je fais de la danse ou du théâtre, ou de l'art... »

7. Clin d'œil discret et approximatif à Pirandello et ses Six personnages en quête d'auteur, à la toute fin de la pièce il s'avère que, en dépit des apparences, les acteurs de Daddy... sont au nombre de six.

8. C'est du moins l'un des arguments développés par Gerard Bester. Un argument dont le contenu est susceptible d'évoluer avec le temps, en fonction de la situation politique en Afrique du Sud. 
9. Pour davantage de précisions sur le parcours et l'esthétique de Robyn Orlin, on pourra se reporter à Olivier Hespel, Robyn Orlin, fantaisiste rebelle, op. cit.

10. Selon la formule employée par Toni Merkel dans Who is Robyn Orlin?

11. Robyn Orlin a d'ailleurs éprouvé comment, lorsqu'elle se trouvait à Londres en 1975, "une Blanche sud-africaine [était] automatiquement perçue comme une proapartheid [...] sa couleur de peau et sa nationalité [étaient] contre elle » (Olivier Hespel, Robyn Orlin, fantaisiste rebelle, op. cit., p. 19).

12. Voie non négligeable, mais qui nous entraînerait trop loin des préoccupations de cet article. Pour rester dans le champ de la danse, on renverra toutefois à Annie Bourdié, "Art chorégraphique contemporain d'Afrique, enjeux d'une reconnaissance ", Marges [En ligne], 16 | 2013, mis en ligne le 15 mars 2014, consulté le 15 août 2020 ; et à Claudia Palazzolo, Mise en scène de la danse aux Expositions de Paris, 1889-1937. Une fabrique du regard, L'Cil d'or, 2017.

13. Propos recueilli le 27 octobre 2011, au CentQuatre, à Paris, sur les répétitions de Have you hugged, kissed and respected your brown Venus today? (Luxembourg, 2011).

14. Sur cette question, cf. Alfred Schütz, L'Étranger. Un essai de psychologie sociale (1944), suivi de L'homme qui rentre au pays (1945), trad. de l'anglais Bruce Bégout, Allia, 2010.

15. Robyn Orlin avec Marie Richeux, dans « Par les temps qui courent ", France Culture, 6/11/2019 (https://www.franceculture.fr/emissions/par-les-temps-qui-courent/robynorlin).

16. Cf. Georges Didi-Huberman, "L'image critique", in Ce que nous voyons, ce qui nous regarde, Minuit, 1992, p. 125.

17. Performance créée pour les Soirées nomades de la Fondation Cartier (Paris), dans le cadre d'une "exposition majeure consacrée à l'artiste César, dix ans après sa disparition " (d'après les archives de la Fondation Cartier pour l'art contemporain, https://www.fondationcartier.com/expositions/cesar-anthologie-par-jean-nouvel).

18. Le "perpétuel » retard de la chorégraphe est désigné par et dans le texte du spectacle; il se manifeste aussi dans le costume lui-même, inachevé, comme le montrent les aiguilles à tricoter qui maintiennent le dernier rang de mailles, au bas de la robe, et la pelote de laine qui lui tient lieu de traîne.

19. Cf. Hannah Arendt, La liberté d'être libre. Les conditions et la signification d'une révolution, trad. Françoise Bouillot, Payot, 2020, en particulier p. 79 et sq.

20. Cf. René Goscinny et Jean Tabary, Le Grand Vizir Iznogoud, Dargaud, 1966.

21. Cf. Franz Kafka, L'Amérique, trad. Alexandre Vialatte, Paris, Gallimard, "folio", 1973, p. 315-341.

22. Hey Dude... Un film de Stéphanie Magnant et Philippe Lainé (13'30, Sweety Pie, 2005).

23. Les allusions et les adresses directes à Robyn-absente sont devenues chez cette artiste des figures de style.

24. Olivier Hespel, Robyn Orlin, Fantaisiste rebelle, op. cit., p. 55.

25. Ibid., p. 56.

26. Cf. Robyn Orlin, de Johannesburg au Palais Garnier, op. cit. Précisons que les situations montrées dans ce film sont vérifiables dans la "réalité » des processus de travail, comme dans les stages dirigés par Robyn Orlin. Ainsi, l'un des exercices d'improvisation 
qu'elle propose à ses interprètes lorsqu'elle entreprend une création consiste à une imaginer une " tâche impossible ».

27. "What you're creating now is your own vocabulary. When you move into something of the known, into the vocabulary of the known, it loses its power. [...] It's the hardest thing in the world, to find a new vocabulary. [...] I like the struggle and that's what interests me. It's interesting to show the struggle and let this struggle become a movement", ibid. (ma traduction).

28. Maurice Blanchot, L'Écriture du désastre, Gallimard, «nrf », 1980, p. 21.

29. Cf. Marie José Mondzain, $K$ comme Kolonie. Kafka et la décolonisation de l'imaginaire, La fabrique éd., 2020, p. 127.

30. Cité par Olivier Hespel, Robyn Orlin fantaisiste rebelle, op. cit., p. 59.

31. La stratégie qui consiste à isoler un spectateur en le montrant du doigt, à laquelle Robyn Orlin a eu recours à diverses reprises, est une forme de stigmatisation. Elle enferme la personne visée dans un rôle, aussi bref soit-il. En cela déjà, elle constitue un raté. Notons également que le retard presque systématique avec lequel le spectateur en question se reconnaît, comprend sa situation, provoque le rire.

32. Ces canards en plastique qui sont eux-mêmes un motif récurrent dans son théâtre.

33. Mais tout de même, dans la mesure où cette histoire de rater concerne aussi la question de la place, nous glisserons là ses trois morales : 1) si on te met dans la merde, ce n'est pas forcément parce qu'on te veut du mal ; 2) si on te sort de la merde, ce n'est pas forcément parce qu'on te veut du bien ; 3) enfin, si tu es dans la merde, quel besoin as-tu de chanter?

\section{RÉSUMÉS}

Partant du principe que, dans les créations scéniques de Robyn Orlin, l'expérience du " rater ", dans ses multiples déclinaisons, fait figure de leitmotiv, on s'efforce de montrer ici, en s'appuyant principalement sur l'analyse de quelques pièces, à quels niveaux et sous quelles formes il se manifeste. Où il s'avère, entre autres, que ce motif a partie liée avec une autre préoccupation centrale dans l'écriture de cette artiste : la question du pouvoir et de son lieu, de ses expressions, de ses manifestations, au théâtre comme dans la société.

\section{INDEX}

Mots-clés : arts de la scène, Orlin (Robyn), dramaturgie, rater 


\section{AUTEUR}

\section{MYRIAM BLCFDE}

Membre de l'équipe THETA (Théories et Histoire de l'Esthétique, du Technique et des Arts) au Centre Jean Pépin (CNRS-ENS, Paris), Myriam Blœdé travaille sur la danse contemporaine envisagée dans ses interactions avec la littérature et les autres arts. Elle a notamment dirigé : Valeska Gert, Je suis une sorcière. Kaléidoscope de ma vie, trad. P. Ivernel, nouv. éd. rev. et augm., L'ÆEil d'or, 2021 ; et compte, parmi ses publications récentes : « Archive dansée / archive de la danse. La démarche historienne d'olga de Soto », Recherches en danse, 7 | 2019 ; « 1929: un anno esplosivofisso. Uno sguardo alle riviste », avec J. Chénieux, in Da Magritte a Duchamp. 1929: il Grande Surrealismo dal Centre Pompidou, Skira 2018 ; « Vu(e)s de Syrie », Images de la Culture, n 30, février 2017 ; « De l'énergie du fauve à la présence des oiseaux. Le bestiaire de Josef Nadj », Ligeia, dossiers sur l'Art, nos 145-148, janvier-juin 2016. 